\title{
Click chemistry approach to ionic liquids (ILs) supported organic synthesis
}

\author{
Alioune Fall ${ }^{1}$, Insa Seck ${ }^{2}$, Massène Sène ${ }^{1}$, Mohamed Gaye ${ }^{1}$, Matar Seck ${ }^{2}$, Generosa Gómez ${ }^{3}$ and \\ Yagamare Fall ${ }^{3, *}$ \\ ${ }^{1}$ Laboratoire de Chimie de Coordination Organique (LCCO), Département de Chimie, Faculté des Sciences et \\ Techniques: Université Cheikh Anta Diop de Dakar, Sénégal. \\ ${ }^{2}$ Département de Chimie, Faculté de Medecine, de Pharmacie et d'Odonto-stomatologie, Université Cheikh Anta \\ Diop, Dakar, Sénégal. \\ ${ }^{3}$ Departamento de Química Orgánica, Facultad de Química and Instituto de Investigación Biomedica (IBI), \\ University of Vigo, Campus Lagoas de Marcosende, 36310 Vigo, Spain
}

\begin{abstract}
A furan substrate anchored to an ionic liquid bound tert-butyldiphenylsiloxane was synthesized using a "click" chemistry approach. The ionic liquid supported furan moiety underwent singlet oxygen oxidation to afford a butenolide intermediate which after removal of the siloxane group gave a bicyclic lactone.
\end{abstract}

Keywords: "Click” chemistry; Ionic liquids; tert-butyldiphenylsiloxane; Singlet oxygen; Butenolide; Lactones.

\section{Introduction}

In the last years, ionic liquids (ILs) have attracted considerable interest as environmentally benign reaction media because of their unique properties such as high thermal and chemical stability, negligible vapour pressure, tunable polarity, nonflammability, friction reduction, antiwear performance, high loading capacity and easy recyclability ${ }^{1}$.

An attractive feature of ionic liquids is that their solubility can be tuned readily. Therefore, phase separation from organic solvent or aqueous phase is allowed depending on the choice of cations and anions. This suggests the possibility of using ILs as soluble support for organic synthesis. Substrates anchored on ionic liquids are expected to retain their reactivity, as in reactions in solution. One advantage of ILs supported synthesis over solid phase synthesis is that conventional spectroscopic analysis can be carried out during the synthetic process. The feasibility of ionic liquid supported organic synthesis has been demonstrated by many research groups ${ }^{2}$.

Silicon protecting groups are of upmost importance in organic synthesis and siliconcontaining linkers are valuable for the attachment of substrates to solid support. Brown and co-workers described the synthesis and applications of tertalkoxysiloxane linkers in solid-phase chemistry ${ }^{3}$. This prompted us to design the synthesis of a tertbutyldiphenylsiloxane linked to an ionic liquid support (Figure 1).

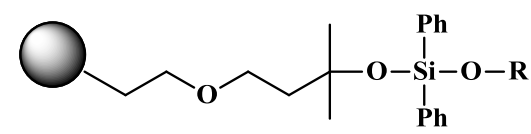

1

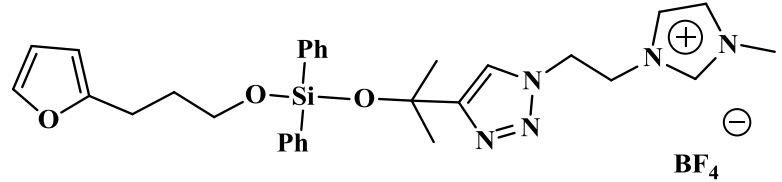

Figure 1. Structures of Brown's resin bound to a tert-butyldiphenylsiloxane linker (1) and the targeted ionic liquid supported tert-butyldiphenylsiloxane linker (2).

\section{Results and Discussion}

As part of our ongoing programme focused on the synthesis of ionic liquids and their application in organic reactions ${ }^{4}$. We now wish to report the synthesis of IL-supported furan 2. Our retrosynthetic basis is outlined in Scheme 1.

*Corresponding author: Yagamare Fall

E-mail address: yagamare@uvigo.es

DOI: http://dx.doi.org/10.13171/mjc.4.4.2015.06.26.11.08/yagamare2 

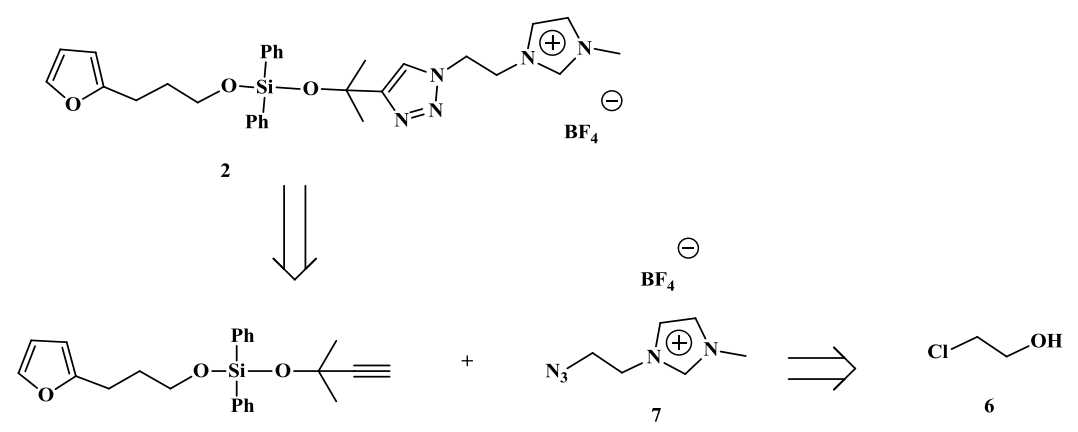

8
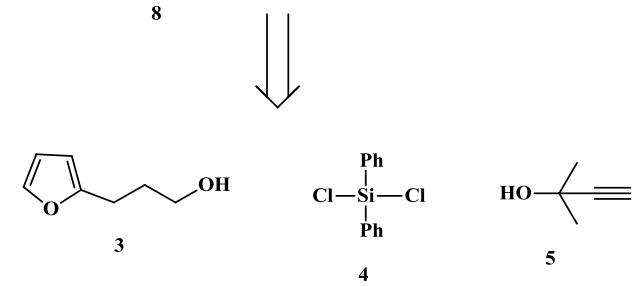

Scheme 1. Retrosynthetic analysis of IL supported furan 2

We anticipated that IL supported furan $\mathbf{2}$ could be obtained using a "Click" chemistry approach ${ }^{5}$

between alkyne 8 and azide $\mathbf{7}$ bearing the IL moiety.

Accordingly compounds $\mathbf{7}$ and $\mathbf{8}$ were prepared as outlined in Scheme 2.

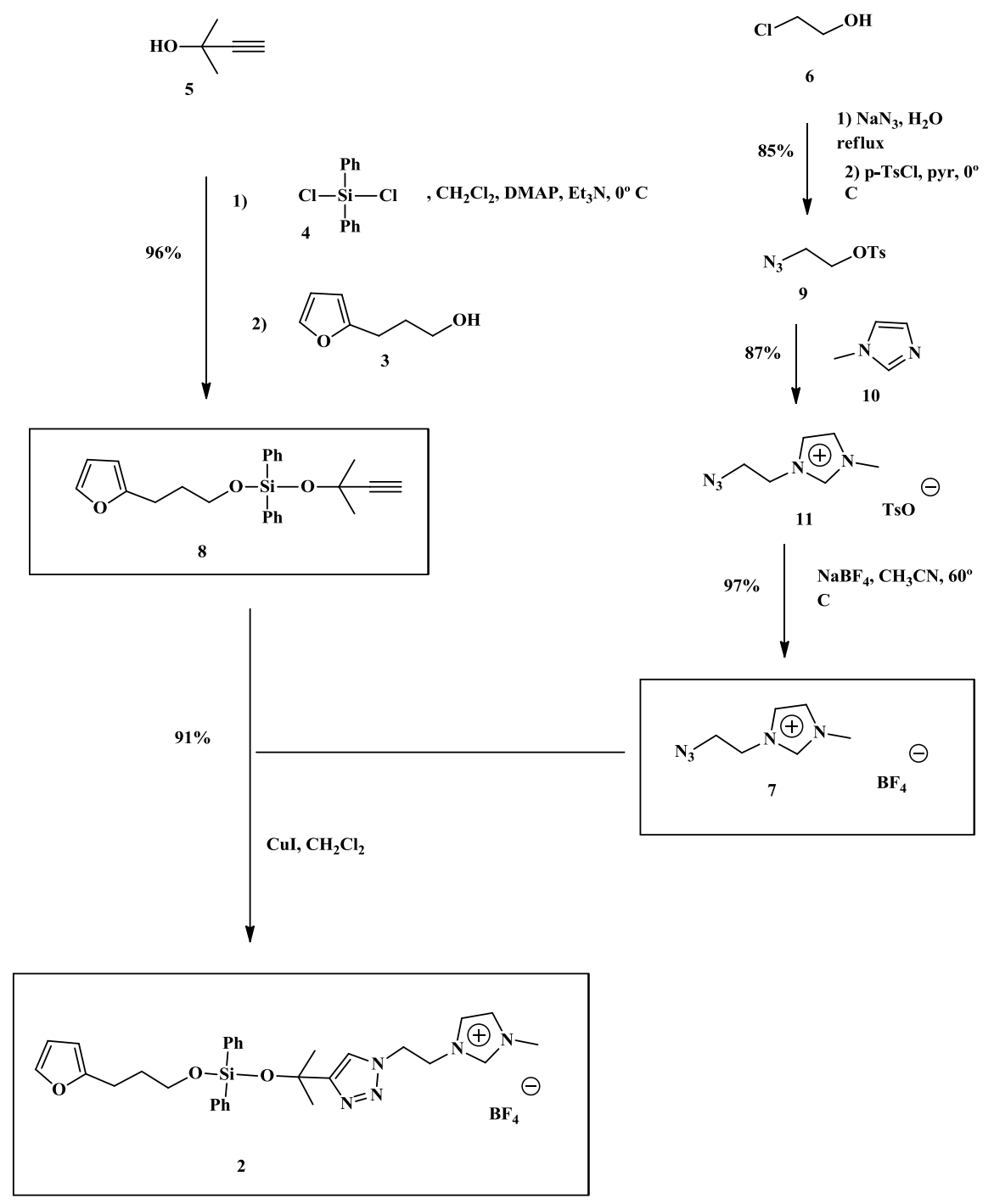

Scheme 2. Synthesis of IL supported furan 2 
Tosylate 9 was easily prepared from commercially available chloride 6 in $85 \%$ overall yield. Quaternization of $\mathbf{9}$ with methyl imidazole $\mathbf{1 0}$ gave $\mathbf{1 1}$ in a $87 \%$ yield. The latter underwent a metathesis reaction to afford the target azide 7 (97\% yield). Compound $\mathbf{8}$ was obtained in a one pot reaction by sequential reaction of propargylic alcohol 5 with dichlorodiphenylsilane $\mathbf{4}$ followed by furan $\mathbf{3}$ to afford the target alkyne $\mathbf{8}$ in an overall yield of $96 \%$. With alkyne 8 and azide 7 in hand, the stage was set for the "click" chemistry reaction which occurred uneventfully giving IL-supported furan $\mathbf{2}$ in $91 \%$ yield.

Our research group developed some years ago a new and original methodology for the synthesis of oxacyclic systems based on the oxidation of a furan ring with singlet oxygen, which we coined "the furan approach ${ }^{6}$. We anticipated that IL-supported furan 2, could undergo a singlet oxygen oxidation to afford methoxy butenolide 12, which after protecting group removal would give bicyclic lactone 13, via an oxa Michael addition (Scheme 3).
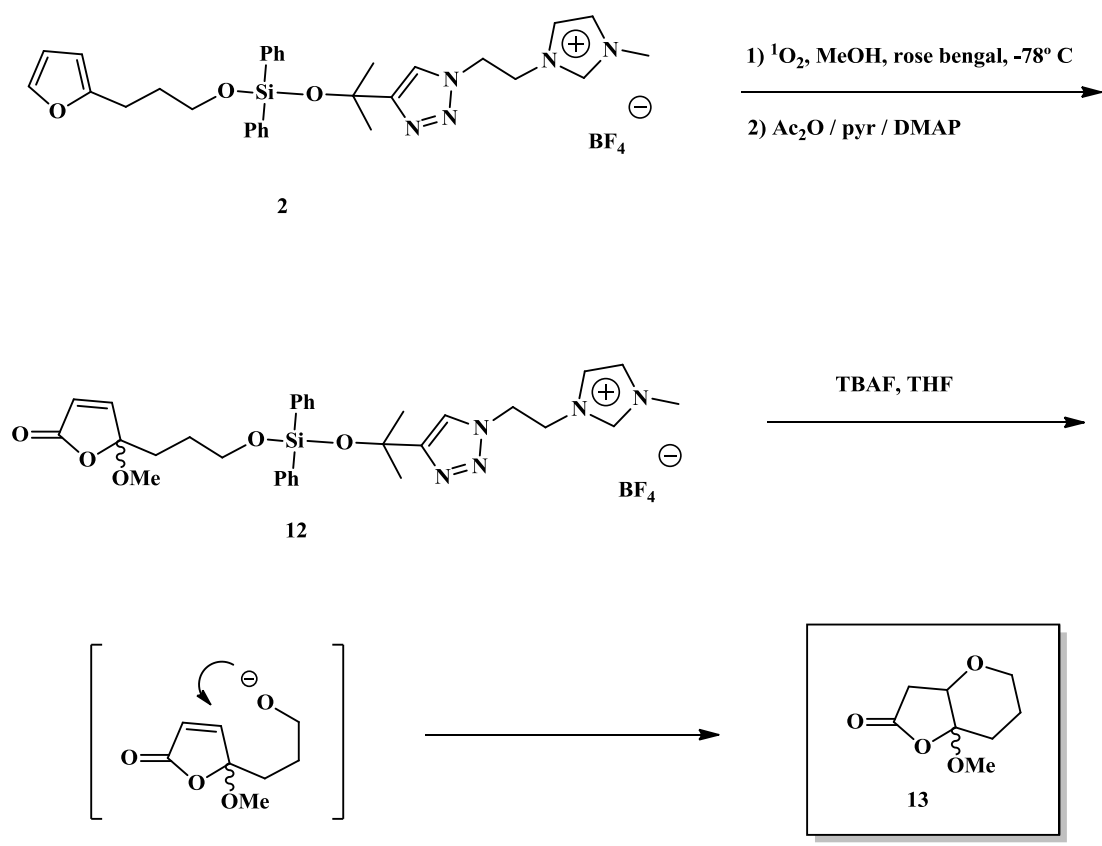

Scheme 3. Synthesis of bicyclic lactone $\mathbf{1 3}$ from IL-supported furan $\mathbf{2}$

Accordingly, singlet oxygen oxidation of compound $\mathbf{2}$ gave butenolide $\mathbf{1 2}$ which was not isolated but treated with TBAF to give the target lactone $\mathbf{1 3}$ in an overall yield of $60 \%$.

\section{Conclusion}

In conclusion we have described a straightforward synthesis of ionic liquid bound tertbutyldiphenylsiloxane. The ionic liquid supported furan moiety underwent singlet oxygen oxidation to afford a butenolide intermediate which after removal of the siloxane group gave a bicyclic lactone. This procedure could be a good alternative to solid-phase synthesis and use of this methodology for ionic liquid supported synthesis of natural products is now under way in our laboratories.

\section{Acknowledgements}

This work was supported financially by the Xunta de Galicia (projects CN 2012/184 and the Galician Network on Ionic Liquids (REGALIs2 (Axuda R2014/015)). The work of the NMR, SC-XRD and MS divisions of the research support services of the
University of Vigo (CACTI) is also gratefully acknowledged. A.F; M.G and M.S. thank the University Cheikh Anta Diop (Dakar) for financial support for a research stay at the University of Vigo. I.S and M.S. thank the Senegalese Ministry of Scientific Research: FIRST (fonds d'impulsion de la recherche scientifique et technique) for a research grant

\section{Experimental Section}

\section{General Procedures}

Solvents were purified and dried by standard procedures. Flash chromatography was performed on silicagel (Merck 60, 230-400 mesh). Analytical TLC was performed on plates precoated with silica gel (Merck 60 F254, $0.25 \mathrm{~mm}$ ). Melting points were obtained using a Gallenkamp apparatus and are uncorrected. Optical rotations were obtained using a Jasco P-2000 polarimeter. IR spectra were obtained using a Jasco FT/IR-6100 Type A spectrometer. ${ }^{1} \mathrm{H}$ NMR (400 MHz) and ${ }^{13} \mathrm{C}$ NMR (100 MHz) spectra were recorded on a Bruker ARX-400 spectrometer using TMS as the internal standard; 
chemical shifts $(\delta)$ are quoted in ppm and coupling constants ( $\mathrm{J}$ ) in Hz. Mass spectrometry (MS and HRMS) was carried out using a Hewlett-Packard 5988A spectrometer. Electrospray mass spectra (ESI-MS) were measured on a Bruker APEXQe FT-ICR MS

2-azidoethyl 4-methylbenzenesulfonate (9).

To a solution of $\mathrm{NaN}_{3} 5.8 \mathrm{~g}$ (89.6 mmol, 2equiv) in water $(70 \mathrm{~mL})$ was added a portionwise $3.00 \mathrm{~mL}$ (44.8 mmol, 1equiv) of 3-chloroethanol (6). The mixture was heated under reflux for $16 \mathrm{~h}$, then cooled to room temperature and extracted with $\mathrm{CH}_{2} \mathrm{Cl}_{2}$ (60 $\mathrm{mL} \times 3$ ). The combined organic phases were dried over $\mathrm{Na}_{2} \mathrm{SO}_{4}$, filtered and evaporated under reduced pressure, affording 3.6 $\mathrm{g}$ of 3 -azidoethanol as a colourless liquid (98\%) used in the next step without any further purification.

2-azidoethanol : $\mathrm{R}_{\mathrm{f}}=0.46($ EtOAc/Hexane $3: 7)$; ${ }^{1} \mathrm{H}-\mathrm{NMR}\left(\mathrm{CDCl}_{3}, \delta\right): 3.67(2 \mathrm{H}, \mathrm{t}, \mathrm{J}=4.84 \mathrm{~Hz}) ; 3.66$ $(1 \mathrm{H}, \mathrm{s}) ; 3.33(2 \mathrm{H}, \mathrm{t}, \mathrm{J}=4.87 \mathrm{~Hz}) ;{ }^{13} \mathrm{C}-\mathrm{NMR}\left(\mathrm{CDCl}_{3}\right.$, $\delta):$ 60.86, 53.04; $\mathrm{MS}\left(\mathrm{EI}^{+}\right)(\mathrm{m} / \mathrm{z}, \%): 83.96$ (100); 85.95 (22); 87.04 (11); 87.95 (8).

To a solution of 2-azidoethanol $(7.8 \mathrm{~g}, 90 \mathrm{mmol})$ in pyridine $(40 \mathrm{~mL})$ was added a portionwise of Tscl $(21 \mathrm{~g} 108 \mathrm{mmol})$ and the mixture was stirred at $0^{\circ} \mathrm{C}$ for $3 \mathrm{~h}$. Water $(60 \mathrm{~mL})$ was added and the product extracted with $\mathrm{Et}_{2} \mathrm{O}(4 \times 80 \mathrm{~mL})$. The combined organic phases were washed with a $10 \%$ aqueous solution of $\mathrm{HCl}$ and brine. After a drying with $\mathrm{Na}_{2} \mathrm{SO}_{4}$, the filtration and solvent evaporation afforded $20 \mathrm{~g}$ of tosylate $9(93 \%)$.

$\mathrm{R}_{\mathrm{f}}=0.53\left(\right.$ EtOAc/Hexane 3:7); ${ }^{1} \mathrm{H}-\mathrm{NMR}\left(\mathrm{CDCl}_{3}, \delta\right)$ : $7.83(2 \mathrm{H}, \mathrm{d}, \mathrm{J}=8.26 \mathrm{~Hz}) ; 7.38(2 \mathrm{H}, \mathrm{d}, \mathrm{J}=8.08 \mathrm{~Hz})$; $4.18(2 \mathrm{H}, \mathrm{t}, \mathrm{J}=5.16 \mathrm{~Hz}) ; 3.50(2 \mathrm{H}, \mathrm{t}, \mathrm{J}=5.04 \mathrm{~Hz})$, $2.48(3 \mathrm{H}, \mathrm{s}) ;{ }^{13} \mathrm{C}-\mathrm{NMR}\left(\mathrm{CDCl}_{3}, \delta\right): 145.26 ; 132.61$; $129.99 ; 127.98 ; 68.04 ; 49.59 ; 21.67$.

\section{Preparation of imidazolium salt 11}

A mixture of tosylate $9(7 \mathrm{~g}, 29 \mathrm{mmol})$ and methyl imidazole $\mathbf{1 0}(2.3 \mathrm{~mL}, 29 \mathrm{mmol})$ was stirred at room temperature for $24 \mathrm{~h}$. The residue was washed with EtOAc ( $3 \times 25 \mathrm{~mL})$ to afford $2 \mathrm{~g}(87 \%)$ of salt 11; ${ }^{1} \mathrm{H}-\mathrm{NMR}\left(\mathrm{CDCl}_{3}, \delta\right): 9.63(1 \mathrm{H}, \mathrm{s}) ; 7.73$ $(2 \mathrm{H}, \mathrm{d}, \mathrm{J}=8.10 \mathrm{~Hz}) ; 7.56(1 \mathrm{H}, \mathrm{s}) ; 7.38(1 \mathrm{H}, \mathrm{s}) ; 7.15$ $(2 \mathrm{H}, \mathrm{d}, \mathrm{J}=8.00 \mathrm{~Hz}) ; 4.39(2 \mathrm{H}, \mathrm{t}, \mathrm{J}=5.51 \mathrm{~Hz}) ; 3.89$ $(3 \mathrm{H}, \mathrm{s}) ; 3.77(2 \mathrm{H}, \mathrm{t}, \mathrm{J}=5.34 \mathrm{~Hz}), 2.34(3 \mathrm{H}, \mathrm{s})$; ${ }^{13} \mathrm{C}-\mathrm{NMR} \quad\left(\mathrm{CDCl}_{3}, \delta\right): 143.68, \quad 169.32,137.59$, $128.78, \quad 125.59,123.55,122.94, \quad 50.30, \quad 48.48$, 36.14,21.21; IR-(CDCl $\left.3, \mathbf{v}\left(\mathrm{cm}^{-1}\right)\right): 2924 ; 2103 ; 1736$; $1575 ; 1450 ; 1349 ; 1289 ; 1192 ; 1122 ; 1034 ; 1011$; 818; 683; 567; MS (ESI) (m/z, \%]: $\mathrm{ESI}^{+} 475.16$ (2cations + 1anion, 10); 152.09 (cation, 100). ESI 494.12 (1cation + 2anions, 100); 171.01 (anion, 99).

\section{Preparation of imidazolium salt 7}

A mixture of imidazolium salt 11 (1 g, 3.4 mmol) and $\mathrm{NaBF}_{4}(0.37 \mathrm{~g}, 3.4 \mathrm{mmoL})$ in $\mathrm{CH}_{3} \mathrm{CN}(20$ $\mathrm{mL}$ ) was stirred at $60^{\circ} \mathrm{C}$ for $24 \mathrm{~h}$. The precipitate was filtered and the organic phase was concentrated to give $0.7 \mathrm{~g}(99 \%)$ of salt 7 .

${ }^{1} \mathrm{H}-\mathrm{NMR}\left(\mathrm{D}_{2} \mathrm{O}, \delta\right): 8.72(1 \mathrm{H}, \mathrm{s}), 7.48(1 \mathrm{H}, \mathrm{s}), 7.41$ $(1 \mathrm{H}, \mathrm{s}), 4.32(2 \mathrm{H}, \mathrm{t}, \mathrm{J}=5.4 \mathrm{~Hz}), 3.85(3 \mathrm{H}, \mathrm{s}), 3.71$ $(2 \mathrm{H}, \mathrm{t}, \mathrm{J}=6.4 \mathrm{~Hz}) ;{ }^{13} \mathrm{C}-\mathrm{RMN}\left(\mathrm{D}_{2} \mathrm{O}, \delta\right): 136.62$,
123.92, 122.56, 50.30, 48.62，36.02，;IR- $\left(\mathrm{CDCl}_{3}\right.$, v( $\left.\left.\mathrm{cm}^{-1}\right)\right): 2924 ; 2853 ; 2360 ; 2105 ; 1576 ; 1455 ; 1351$; $1292 ; 1168 ; 1055 ; 770 ; 649 ; 622 ; 521$; MS (ESI) (m/z, \%]: ESI $^{+} 391.18$ (2cations + 1anion, 18); 152.09 (cation, 100). ESI 649.20 (2cations + 4anions, 39); 565.19 (2cations + 3anions, 100); 326.10 (1cation + 2anions, 70); 171.01 (2anions, 47).

\section{Preparation of compound 8}

To a solution of propargylic alcohol $5(0.5 \mathrm{~mL}$, $5.2 \mathrm{mmol})$ in $\mathrm{CH}_{2} \mathrm{Cl}_{2}(10 \mathrm{~mL})$ at $0^{\circ} \mathrm{C}$ were added $\mathrm{Et}_{3} \mathrm{~N}$ (1.12 mL, $\left.15.5 \mathrm{mmol}\right)$, dichlorodiphenylsilane $4(1.1 \mathrm{~mL}, 5.2 \mathrm{mmol})$ and a catalytic amount of DMAP (monitoring the course of the reaction by tlc). The organic solvent was concentrated and water $(20 \mathrm{~mL})$ was added. The product was extracted with $\mathrm{Et}_{2} \mathrm{O}(2 \times 15 \mathrm{~mL})$. The combined organic phases were washed with brine $(2 \times 15 \mathrm{~mL})$, dried over $\mathrm{Na}_{2} \mathrm{SO}_{4}$. Filtration and solvent evaporation afforded a residue which was chromatographed on silica using $30 \%$ EtOAc / Hexane as eluent, affording $3.7 \mathrm{~g}(96 \%$ yield) of alkyne $\mathbf{8}$ as a colourless oil. Rf: 0,82 (30\% EtOAc/Hexane); ${ }^{1} \mathrm{H}-\mathrm{NMR}\left(\mathrm{CDCl}_{3}, \delta\right): 7.69(4 \mathrm{H}$, dd, $\mathrm{J}=2.8 \mathrm{~Hz}, \mathrm{~J}=1.6 \mathrm{~Hz}) ; 7.39(6 \mathrm{H}, \mathrm{m}) ; 7.29(1 \mathrm{H}, \mathrm{m})$; $6.22(1 \mathrm{H}, \mathrm{dd}, \mathrm{J}=2.9 \mathrm{~Hz}, \mathrm{~J}=1.7 \mathrm{~Hz}) ; 5.91(1 \mathrm{H}, \mathrm{dd}$, $\mathrm{J}=2.6 \mathrm{~Hz}, \mathrm{~J}=2.00 \mathrm{~Hz}) ; 3.83(2 \mathrm{H}, \mathrm{m},) ; 2.75(2 \mathrm{H}, \mathrm{t}$, $\mathrm{J}=7.7 \mathrm{~Hz}) ; 2.29(1 \mathrm{H}, \mathrm{s}) ; 1.95(2 \mathrm{H}, \mathrm{m}) ; 1.53(6 \mathrm{H}, \mathrm{s})$; ${ }^{13} \mathrm{C}-\mathrm{NMR} \quad\left(\mathrm{CDCl}_{3}, \delta\right): 155.86,140.70,135.36$, $134.14,129.74,127.57,110.02,104.82,88.37$, 70.69, 67.91, 62.29, 32.53, 30.53, 24.35; IR-(CDCl ${ }_{3}$, $\left.v\left(\mathrm{~cm}^{-1}\right)\right): 2984,2935,2874,1591,1507,1429,1380$, 1361, 1226, 1151, 1116, 1095, 1046, 1007, 799, 739, 718, 700, 524; MS $\left(\mathrm{EI}^{+}\right)(\mathrm{m} / \mathrm{z}, \%) 413.15([\mathrm{M}+$ $\left.\mathrm{Na}]^{+}, \quad 38\right) ; 391.17\left([\mathrm{M}+\mathrm{H}]^{+}, \quad 100\right) ; 325.13$ $\left(\left[\mathrm{C}_{19} \mathrm{H}_{20} \mathrm{O}_{3} \mathrm{Si}\right]^{+}, \quad 72\right) ; 265.09 \quad\left(\left[\mathrm{C}_{17} \mathrm{H}_{17} \mathrm{OSi}\right]^{+}, \quad 21\right)$; $136.06\left(\left[\mathrm{C}_{6} \mathrm{H}_{4} \mathrm{O}_{2} \mathrm{Si}\right]+, 37\right)$; HRMS $\left(\mathrm{EI}^{+}\right): 390.1621$ calcd for $\mathrm{C}_{24} \mathrm{H}_{26} \mathrm{O}_{3} \mathrm{Si}$; found: 307.1651 .

\section{Preparation of IL supported furan 2}

To a solution of azide 7 (423.4 $\mathrm{mg}, 1.44 \mathrm{mmol})$ in $\mathrm{CH}_{2} \mathrm{Cl}_{2}(3 \mathrm{~mL})$ were added alkyne $\mathbf{8}(609 \mathrm{mg}$, $1.56 \mathrm{mmol})$ and $\mathrm{CuI}(27.5 \mathrm{mg}, 0.14 \mathrm{mmol})$. The mixture was stirred at room temperature for $48 \mathrm{~h}$ and the solvent concentrated. Ether was added to the residue and the organic phase was decanted, in order to remove excess of starting alkyne. $\mathrm{CH}_{2} \mathrm{Cl}_{2}(100$ $\mathrm{mL}$ ) was added and the solution was filtered through a short pad of silicagel. Solvent evaporation afforded $899 \mathrm{mg}$ of $2(91 \%)$ as a solid. M.p: $77-79^{\circ} \mathrm{C}$;

${ }^{1} \mathrm{H}-\mathrm{NMR}\left(\mathrm{CDCl}_{3}, \delta\right): 8.70(1 \mathrm{H}, \mathrm{s}), 7.58-7.56(4 \mathrm{H}$, $\mathrm{m}), 7.56(1 \mathrm{H}, \mathrm{s}), 7.36-7.28(6 \mathrm{H}, \mathrm{m}), 6.96(2 \mathrm{H}, \mathrm{d}$, $\mathrm{J}=1.25 \mathrm{~Hz}), 6.23(1 \mathrm{H}, \mathrm{dd}, \mathrm{J}=1.88 \mathrm{~Hz}, \mathrm{~J}=1.92 \mathrm{~Hz})$; $5.89(1 \mathrm{H}, \mathrm{dd}, \mathrm{J}=1.72 \mathrm{~Hz}, \mathrm{~J}=2.00 \mathrm{~Hz}) ; 4.72(4 \mathrm{H}, \mathrm{s})$, $3.77(3 \mathrm{H}, \mathrm{s}), 3.74(2 \mathrm{H}, \mathrm{t}, \mathrm{J}=6.20 \mathrm{~Hz}), 2.69(2 \mathrm{H}, \mathrm{t}$, $\mathrm{J}=7.52 \mathrm{~Hz}), 1.85(1 \mathrm{H}, \mathrm{m}), 1.65(6 \mathrm{H}, \mathrm{s}) ;{ }^{13} \mathrm{C}-\mathrm{NMR}$ $\left(\mathrm{CDCl}_{3}, \quad \delta\right): \quad 156.10,155.72,140.77,137.35,134.86$, 134.81,134.56, 134.32,134.28,134.01,130.25,130.06, 127.91.127.83,127.76,127.73,122.91,

$122.73,121.75,110.05,104.87,72.49,62.19,48.97,48.8$ 8, 36.31, 30.91, 30.54, 24.31; MS (ESI) (m/z, \%): $\mathrm{ESI}^{+} 542.26$ (cation, 100). ESI' 1061.82 (33); 884.29 
(cation, 2anions, 88); 748.25 (cation +2 anions); 524.52 (47); 357.72 (28); 131.54 (100).

7a-methoxyhexahydro-2H-furo[3,2-b]pyran2-one (13).

To a solution of furan $2(599 \mathrm{mg}, 0.84 \mathrm{mmol})$ in dry methanol $(12 \mathrm{~mL})$ was added Rose bengal $(17 \mathrm{mg})$. The mixture was purged several times with $\mathrm{O}_{2}$ (balloon), cooled to $-78^{\circ} \mathrm{C}$ and irradiated with a $200 \mathrm{~W}$ lamp for $3 \mathrm{~h}$, stirring under oxygen atmosphere. The mixture was allowed to reach room temperature and the solvent was evaporated. After solvent evaporation the residue was dissolved in pyridine $(5 \mathrm{~mL})$, acetic anhydride $(1.5 \mathrm{~mL})$ then DMAP (catalytic) were added and the mixture stirred at room temperature overnight. $\mathrm{MeOH}(5 \mathrm{~mL})$ was added and stirring continued for $30 \mathrm{~min}$. The methanol was rotatory evaporated. The residue was treated with $\mathrm{H}_{2} \mathrm{O}(20 \mathrm{~mL})$ and the product extracted with $\mathrm{CH}_{2} \mathrm{Cl}_{2}(3 \times 15 \mathrm{~mL})$. The combined organic phases were washed with a $10 \%$ aqueous solution of $\mathrm{CuSO}_{4}(2 \times 15 \mathrm{~mL})$, dried over $\mathrm{Na}_{2} \mathrm{SO}_{4}$ and the solvent concentrated giving a residue which was dissolved in THF $(15 \mathrm{~mL})$. Tetrabutylammonium fluoride $(0.84 \mathrm{~mL}$ of $1.0 \mathrm{M}$ solution in THF, 0.84 mmol) was added and the mixture was stirred at room temperature for $4 \mathrm{~h}$. Aqueous saturated solution of $\mathrm{NaHCO}_{3}(30 \mathrm{~mL})$ was added and the product extracted with ethyl acetate $(3 \times 20 \mathrm{~mL})$. The combined organic phases were washed with brine (2 x $15 \mathrm{~mL}$ ), dried over $\mathrm{Na}_{2} \mathrm{SO}_{4}$, filtered and the solvent evaporated giving a residue which was chromatographed on silica using 20\% EtOAc / Hexane as eluent, affording $87 \mathrm{mg}(60 \%$ overall yield) of bicyclic lactone 13; Rf: $0,45 \quad(30 \%$ EtOAc/Hexane); ${ }^{1} \mathrm{H}-\mathrm{NMR}\left(\mathrm{CDCl}_{3}, \delta\right): 3,88(1 \mathrm{H}, \mathrm{d}$, $J=4,4 \mathrm{~Hz}, \mathrm{CH}-1) ; 3, .85(1 \mathrm{H}, \mathrm{m}, \mathrm{CH}-3) ; 3,37(1 \mathrm{H}, \mathrm{dd}$, $J=11,7 \mathrm{~Hz}, J=1,7 \mathrm{~Hz}, \mathrm{CH}-3) ; 3,33\left(3 \mathrm{H}, \mathrm{s}, \mathrm{OCH}_{3}\right)$; $2,88(1 \mathrm{H}, \mathrm{dd}, J=17,2 \mathrm{~Hz}, J=4,4 \mathrm{~Hz}, \mathrm{CH}-9) ; 2,52(1 \mathrm{H}$, m, CH-5); 2,33 (1H, d, J=17,2 Hz, CH-9); 1,75 (1H, $\mathrm{m}, \mathrm{CH}-5) ; 1,66(2 \mathrm{H}, \mathrm{m}, \mathrm{CH}-4) ;{ }^{13} \mathrm{C}-\mathrm{NMR}\left(\mathrm{CDCl}_{3}\right.$, $\delta):$ 176,00 (CO); 104,61 (C-6); 76,48 (CH-1); 65,39 $\left(\mathrm{CH}_{2}-3\right) ; 49, .35\left(\mathrm{CH}_{3} \mathrm{O}\right) ; 37,02\left(\mathrm{CH}_{2}-9\right) ; 27,16\left(\mathrm{CH}_{2}-\right.$ 5); 21,52 $\left(\mathrm{CH}_{2}-4\right) ; \mathrm{MS}\left(\mathrm{EI}^{+}\right)[\mathrm{m} / \mathrm{z},(\%)]: 141,05$ $\left(\mathrm{M}^{+}-\mathrm{OMe}, 41\right) ; 123,04$ (22), 113,06 (8); 102,06 (8); 101,05 (100); 100,05 (10); 99,04 (29); 97,06 (36); 72,06 (8); 71,04 (10); 69,03 (13); HRMS (EI+ ${ }^{+}$: calcd for $\mathrm{C}_{8} \mathrm{H}_{12} \mathrm{O}_{4}, 172,0736$; found: 172,0743.

\section{References}

1 - For recent reviews, see (a) T. Welton Chem. Rev. 1999, 99, 2071-2083. (b) P. Wasserscheid, W. Keim, Angew. Chem., Int. Ed. 2000, 39, 3772-3789; (c) J. S. Wilkes Green Chem. 2002, 4, 73-80; (d) J. Dupont, R. F. de Souza, P. A. Z. Suarez, Chem. Rev. 2002, 102, 3667-3691;

(e) P. Wasserscheid, T. Welton Ionic Liquids in Synthesis; Wiley-VCH: Weinheim, Germany, 2003.

2 - (a) J-Y. Huang, M. Lei, Y-G. Wang, Tetrahedron Lett. 2006, 47, 3047-3050 and referencies therein. (b) W. Miao, T. H. Chan, Acc. Chem. Res. 2006, 39, 897-908.

3 - M. M. Meloni, P. D. White, D. Armour, R. C. D. Brown Tetrahedron 2007, 63, 299-311.

4 - (a) A. Fall, M. Sène, M. Gaye, G. Gómez, Y. Fall, Tetrahedron Lett. 2010, 51, 4501-4504; (b) A. Fall, M. Sène, E. Tojo, G. Gómez, Y. Fall, Synthesis 2010, 20, 3415-3417; (c) A. Fall, M. Sène, O. Diouf, M. Gaye, G. Gómez, Y. Fall, Open Org. Chem.J. 2012, 6, 21-26.

5 - (a) H. C. Kolb, M. G. Finn, K. B. Sharpless Angew. Chem., Int. Ed. 2001, 40, 2004-2021; (b) V. V. Rostovtsev, L. G. Green, V. V. Fokin, K. B. Sharpless, Angew. Chem., Int. Ed. 2002, 41, 2596-2599; (c) C. W. Toroe, M. Christensen, M. Meldal J. Org. Chem. 2002, 67, 3057-3064; (d) A. Krasiñski, V. V. Forkin, K. B. Sharpless Org. Lett. 2004, 6, 1237-1240; (e) T. Thirumurugan, D. Matosiuk, K. Jozwiak Chem. Rev. 2013, 113, 4905-4979.

6 - (a) Y. Fall, B. Vidal, D. Alonso, G. Gómez Tetrahedron Lett. 2003, 44, 4467-4469; (b) M. Pérez, P. Canoa, G. Gómez, C. Terán, Y. Fall Tetrahedron Lett. 2004, 45, 5207-5209; (c) D. Alonso, M. Pérez, G. Gómez, B. Covelo, Y. Fall Tetrahedron. 2005, 61, 2021-2026; (d) M. Teijeira, P. L. Suárez, G. Gómez, C. Terán, Y. Fall Tetrahedron Lett. 2005, 46, 5889-5892; (e) I. García, G. Gómez, M. Teijeira, C. Terán, Y. Fall Tetrahedron Lett. 2006, 47, 1333-1335; (f) P. Canoa, M. Pérez, B. Covelo, G. Gómez, Y. Fall Tetrahedron Lett. 2007, 48, 3441-3443; (g) C. Álvarez, M. Pérez, A. Zúñiga, G. Gómez, Y. Fall Synthesis 2010, 22, 3883-3890; (h) P. Canoa, Z. Gándara, M. Pérez, R. Gago, G. Gómez, Y. Fall Synthesis 2011, 3, 431-436; (h) M. Gónzalez, Z. Gándara, B. Covelo, G. Gómez, Y. Fall Tetrahedron Lett. 2011, 52, 5983-5986; (i)A. Zúñiga, G. Pazos, P. Besada, Y. Fall Tetrahedron Lett. 2012, 53, 4293-4295; (j) M. González, Z. Gándara, G. Pazos, G. Gómez, Y. Fall Synthesis 2013, 45, 625-632; (k) M. Gónzalez, Z. Gándara, A. Martínez, G. Gómez, Y. Fall Tetrahedron Lett. 2013, 54, 3647-3650; (1) M. Gónzalez, Z. Gándara, A. Martínez, G. Gómez, Y. Fall Synthesis 2013, 45, 1693-1700; M. González, Z. Gándara, M. Seck, G. Gómez, Y. Fall Mediterr. J. Chem. 2015, 4(3), 18-29. 